\title{
Stimulatory effect of dexamethasone on angiotensin-converting enzyme in neonatal rat cardiac myocytes
}

\section{M.L.M. Barreto-Chaves'1,2, \\ A. Heimann ${ }^{1}$ \\ and J.E. Krieger ${ }^{1}$}

\author{
${ }^{1}$ Laboratório de Genética e Cardiologia Molecular, Instituto do Coração, \\ Faculdade de Medicina, and ${ }^{2} D$ epartamento de Anatomia, \\ Instituto de Ciências Biomédicas, Universidade de São Paulo, São Paulo, SP, Brasil
}

\begin{abstract}
\section{Correspondence}

J.E. Krieger

Laboratório de Genética e

Cardiologia Molecular

INCOR, HC, FM, USP

05403-000 São Paulo, SP

Brasil

Fax: +55-11-3069-5022

E-mail: krieger@ incor.usp.br

Presented at the III International Symposium on Vasoactive Peptides, Belo Horizonte, MG, Brasil, October 8-10, 1999.

Research supported by FAPESP (No. 96/08546-5).

Angiotensin-converting enzyme (ACE) plays a central role in cardiac remodeling associated with pathological conditions such as myocardial infarction. The existence of different cell types in the heart expressing components of the renin-angiotensin system makes it difficult to evaluate their relative role under physiological and pathological conditions. Since myocytes are the predominant cellular constituent of the heart by mass, in the present study we studied the effects of glucocorticoids on ACE activity using well-defined cultures of neonatal rat cardiac myocytes. Under steady-state conditions, ACE activity was present at very low levels, but after dexamethasone treatment ACE activity increased significantly (100 nmol/l after $24 \mathrm{~h})$ in a time-dependent fashion. These results demonstrate the influence of dexamethasone on ACE activity in rat cardiac myocytes. This is consistent with the idea that ACE activation occurs under stress conditions, such as myocardial infarction, in which glucocorticoid levels may increase approximately 50 -fold.
\end{abstract}

Received November 26, 1999 Accepted April 7, 2000

\section{Key words}

- Dexamethasone

- Cardiac myocytes

- Angiotensin-converting

enzyme
The renin-angiotensin system plays a critically important role in the control of cardiovascular and renal homeostasis. There is accumulating evidence for the existence of an independent tissue (local) renin-angiotensin system in several organs, including the heart. The intracardiac renin-angiotensin system is thought to be involved in the development of cardiac hypertrophy, since Ang II itself is a stimulus for growth. In vitro, Ang II induces not only mRNA expression of the protooncogenes c-fos, c-myc, and c-jun in cardiac myocytes and fibroblasts (1), but also hypertrophy and/or hyperplasia of these cells $(1,2)$. Experimental studies on pressure-overloaded left ventricular hypertrophy and experimental heart failure have shown the induction of angiotensin-converting enzyme (ACE) activity and ACE mRNA synthesis (3). Thus, the functions of cardiac ACE go beyond blood pressure control and may be associated with hypertrophy, fibrosis, and the inflammatory response (4). Putative cells that may contribute to local activation of ACE are endothelial cells, myocytes, fibroblasts and monocytes, although the cells responsible for ACE induction associated with these diseases have not yet been clearly identified.

Various hormones have been reported to induce ACE production and release by dif- 
Figure 1 - Neonatal rat cardiomyocytes in culture. Immunofluorescence shows cells stained with anti-tropomyosin (sarcomeric) antibody, which reacts specifically with striated muscle. Magnification 100X (top) and 200X (bottom).
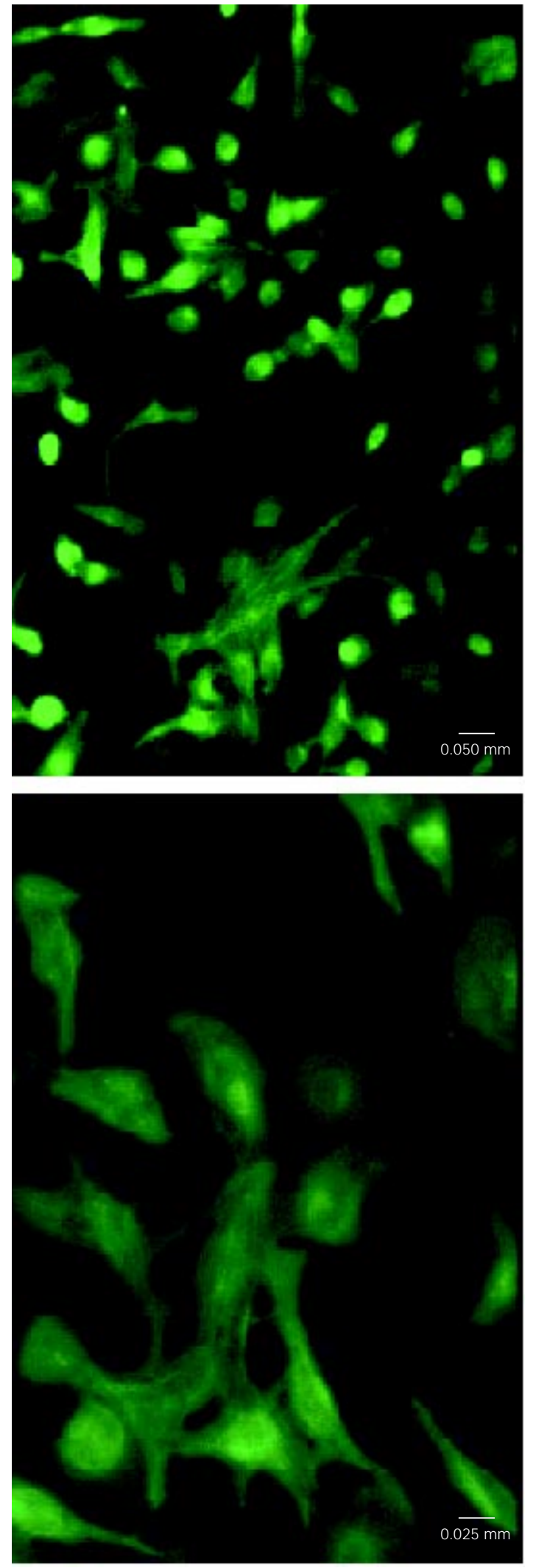

ferent cell types in culture. For instance, glucocorticoids promote the increase of both Ang II formation and bradykinin degradation in vascular smooth muscle (5). Interestingly, high cortisol levels have been frequently found in patients with acute myocardial infarction or acute coronary insufficiency (6).

In view of the growth-promoting properties of Ang II on the myocardium and the action of glucocorticoids on ACE activity in diverse cells under normal and pathological conditions, we have investigated the regulation of the enzyme in well-defined primary cultures of neonatal rat cardiac myocytes after dexamethasone treatment.

Cardiac myocytes were prepared by enzymatic disaggregation as described by Kim et al. (7). Briefly, hearts from neonatal (1-3 days old) Wistar rats were excised and ventricles were minced and transferred to a sterile buffer. The tissue was then subjected to multiple enzymatic digestions at $37^{\circ} \mathrm{C}$ using a mixture of collagenase and pancreatin. Four to five subsequent digestions, each lasting a period of $20 \mathrm{~min}$, were performed. The solution obtained in each digest was transferred to a tube containing $1 \mathrm{ml}$ newborn calf serum (NCS) and centrifuged. Each cell pellet was resuspended in NCS. Dissociated cells were pooled. To separate myocytes from non-myocytes, this cell suspension was layered onto discontinuous Percoll density gradients consisting of two phases. After washing to remove all traces of Percoll, myocytes were cultured in DMEM containing $5 \%$ fetal calf serum, $10 \%$ horse serum and bromodeoxyuridine $(100 \mu \mathrm{M})$. After $24 \mathrm{~h}$, myocytes were cultured in serum-free media.

The purity of cardiac myocytes in culture was evaluated by immunofluorescence in cells seeded on glass cover slips and maintained in serum-free media for at least $24 \mathrm{~h}$. Double immunofluorescence staining using a monoclonal antibody against vimentin (Sigma Chemical Co., St. Louis, MO, USA) 
and against sarcomeric tropomyosin (Sigma) was used. The preparations were mounted and observed under a confocal microscope.

Using this methodology, more than $90 \%$ of cardiomyocytes in culture were visualized by immunohistochemistry (Figure 1).

Dexamethasone (Sigma) was prepared as a stock solution of $10 \mathrm{mg} / \mathrm{ml}$ in absolute ethanol. Cells were growth arrested for $48 \mathrm{~h}$ in serum-free media prior to glucocorticoid treatment.

ACE activity was determined by a fluorimetric assay based on the rate of generation of His-Leu by hydrolysis of hippuryl-HisLeu substrate (Sigma) (8).

The basal ACE activity in cardiac myocyte cells was very low (0.4 nmol His-Leu $\min ^{-1} \mathrm{mg}$ protein $\left.{ }^{-1}\right)$. The addition of dexamethasone $(1000 \mathrm{nmol} / \mathrm{l})$ to the culture medium resulted in an increase in ACE activity (Figure 2A). Enzymatic activity increased after $48 \mathrm{~h}$ of treatment with dexamethasone (100 nmol/l). Furthermore, cells were also incubated with $100 \mathrm{nmol} / \mathrm{l}$ of dexamethasone for different periods of time before harvesting. ACE activity increased significantly at $72 \mathrm{~h}$ of treatment and reached a peak of 3 times the basal levels (Figure 2B). Dexamethasone had no effect on protein concentration.

Our data demonstrate for the first time that glucocorticoid treatment can induce ACE activity in cardiac myocytes. It is tempting to speculate that a similar response can occur in vivo under stress. Although we recognize the limitations of extrapolating results obtained under controlled experimental conditions, it is important to emphasize that glucocorticoid levels (predominantly cortisol) may
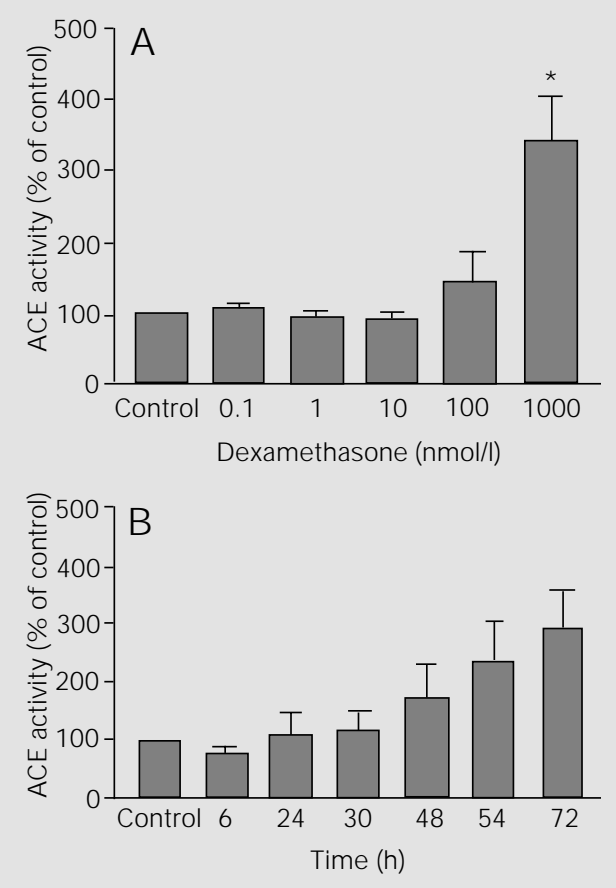

increase 50-fold in human plasma during stress such as surgery and extreme athletic competition $(9,10)$. Thus, it is conceivable that under particularly conditions cells may be exposed to glucocorticoid concentrations that approximate those used in the present experiments. Sustained elevation of glucocorticoids, as occurs in disease states such as Cushing's syndrome, is associated with hypertension and vascular damage. In addition, acute myocardial infarction is a situation in which serum cortisol levels are increased (6). Taken together, the present results demonstrate that ACE activity in rat cardiac myocytes, which represent a major component of cardiac mass, is influenced by glucocorticoid treatment.
Figure 2-A, Effect of dexamethasone on angiotensin-converting enzyme (ACE) activity of neonatal rat cardiac myocytes. ACE activity was measured after $48 \mathrm{~h}$ of culture in the presence or absence (control) of dexamethasone. Results are reported as percent of control (means \pm SD for 4 determinations). $* \mathrm{P}<0.05$ compared to control (one-way ANOVA). B, Bar graph showing the time course of the effect of dexamethasone on ACE activity. Cardiac myocytes were incubated for the indicated time periods with $100 \mathrm{nmol} / \mathrm{l}$ dexamethasone. Results are expressed as percent of the control (mean \pm SD of four independent experiments).

\section{References}

1. SadoshimaJ \& Izumo S (1993). Molecular characterization of angiotensin II-induced hypertrophy of cardiac myocytes and hyperplasia of cardiac fibroblasts. Critical role of the AT1 receptor subtype. Circulation Research, 73: 413-423.
2. Baker KM \& Aceto J F (1990). Angiotensin II stimulation of protein synthesis and cell growth in chick heart cells. American J ournal of Physiology, 259: H610-H618.

3. Hirsch $A$, Talsness $C$, Schunkert $H$, Paul M \& Dzau V (1991). Tissue-specific activa- tion of cardiac angiotensin converting enzyme in experimental heart failure. Circulation Research, 69: 475-482.

4. Falkenhahn $M$, Franke $F$, Bohle RM, Zhu $Y$, Stauss HM, Bachmann S, Danilov S \& Unger T (1995). Cellular distribution of an- 
giotensin-converting enzyme after myocardial infarction. Hypertension, 25: 219226.

5. Fishel RS, Eisenberg S, Shai SY, Redden RA, Bernstein KE \& Berk BC (1995). Glucocorticoids induce angiotensin-converting enzyme expression in vascular smooth muscle. Hypertension, 25: 343349.

6. Kahana L, Keidar S, Sheinfeld M \& Palant A (1983). Endogenous cortisol and thyroid hormone levels in patients with acute myocardial infarction. Clinical Endocrinology, 19: 131-139.

7. Kim NN, Vilarreal FJ , Printz MP, Lee AA \& Dillmann WH (1995). Trophic effects of angiotensin II on neonatal rat cardiac myocytes are mediated by cardiac fibroblasts. American J ournal of Physiology, 269: E426-E437.

8. Oliveira EM, Santos R \& Krieger J E (2000). Standardization of the fluorimetric assay to determine tissue ACE activity in rats. Brazilian J ournal of Medical and Biological
Research (in press).

9. Czeisler CA, Moore MC, Regenstein QR, Kisch ES, Fang VS \& Ehrlich EN (1976). Episodic 24-hour cortisol secretion in patients awaiting elective cardiac surgery. J ournal of Clinical Endocrinology and Metabolism, 42: 273-283.

10. Sutton J R \& Casey J H (1975). The adrenocortical response to competitive athletics in veteran athletes. J ournal of Clinical Endocrinology and Metabolism, 40: 135-138. 